\title{
Effect of sevoflurane on grafted kidney function in renal transplantation
}

\author{
Jin Ha Park ${ }^{1}$, Jae Hoon Lee ${ }^{1,2}$, Dong Jin Joo ${ }^{3}$, Ki Jun Song ${ }^{4}$, Yu Seun Kim ${ }^{3}$, and Bon-Nyeo Koo ${ }^{1,2}$ \\ ${ }^{1}$ Department of Anesthesiology and Pain Medicine, ${ }^{2}$ Anesthesia and Pain Research Institute, Yonsei University College of Medicine, \\ Departments of ${ }^{3}$ Surgery, ${ }^{4}$ Biostatistics, Yonsei University College of Medicine, Seoul, Korea
}

Background: The objective of this retrospective study was to determine if there are any differences in grafted kidney function in recipients of kidney transplantation (KT) when donors and recipients were anesthetized with sevoflurane compared to desflurane.

Methods: Seventy-three pairs of donors-recipients were anesthetized with sevoflurane (Sevo group) and 71 pairs were anesthetized with desflurane (Des group). We retrospectively investigated the blood urea nitrogen (BUN) levels, creatinine (Cr) levels, and estimated glomerular filtration rates (eGFR) of the recipients in both groups for 1 year postoperatively. We tested non-inferiority for serum creatinine at discharge and 1 year after KT. Short-term (1 year) outcomes of KT were assessed by the incidence of delayed graft function (DGF), acute rejection episodes (ARE), and graft failure.

Results: There were no differences in BUN, Cr, eGFR, or outcomes of KT at 1 year postoperatively. Specifically, the 95\% confidence interval for the difference in creatinine levels between the Sevo and Des groups was less than the margin of equivalence at the time of discharge and 1 year after surgery. The occurrences of DGF, ARE, and graft failure were comparable between the groups.

Conclusions: Compared to desflurane, sevoflurane had no adverse effects on grafted renal function or on the shortterm outcome of renal transplantation. (Korean J Anesthesiol 2012; 62: 529-535)

Key Words: Creatinine, Glomerular filtration rate, Kidney transplantation, Sevoflurane.

\footnotetext{
Received: October 14, 2011. Revised: November 15, 2011. Accepted: November 28, 2011.

Corresponding author: Bon-Nyeo Koo, M.D., Ph.D., Department of Anesthesiology and Pain Medicine, Anesthesia and Pain Research Institute, Yonsei University College of Medicine, 50, Yonsei-ro, Seodaemun-gu, Seoul, Korea. Tel: 82-2-2228-2420, Fax: 82-2-2227-7897, E-mail: koobn@yuhs.ac (c) This is an open-access article distributed under the terms of the Creative Commons Attribution Non-Commercial License (http:// creativecommons.org/licenses/by-nc/3.0/), which permits unrestricted non-commercial use, distribution, and reproduction in any medium, provided the original work is properly cited.
} 


\section{Introduction}

Currently, kidney transplantation (KT) is the treatment of choice for patients with end-stage renal failure. The transplantation surgery is commonly performed under general anesthesia using a volatile anesthetic agent. However, sevoflurane - a commonly used volatile anesthetic - has the potential to adversely affect the function of a grafted kidney as well as the outcome of KT because an inorganic fluoride ion produced during sevoflurane metabolism is related to methoxyfluraneinduced nephrotoxicity [1]. Furthermore, compound A - another product of sevoflurane metabolism in the presence of carbon dioxide - can affect the breathing circuit and has been shown to cause renal damage in animals [2]. Although it has been generally accepted that sevoflurane can be used without concern for nephrotoxicity [3], this is the subject of a debate that is still ongoing because of a number of reports of sevofluraneinduced nephrotoxicity in humans [4-8].

Furthermore, grafted kidneys are very sensitive during transplantation. Various factors, such as ischemia-reperfusion (IR) injury, can cause early renal dysfunction, which can increase the risk of acute rejection episodes and graft failure $[9,10]$. Reports regarding the effects of sevoflurane on transplanted kidneys are limited, although there have been several suggestions of increased risk of renal damage while using sevoflurane [11].

Therefore, the objective of this retrospective study was to determine whether there are any differences in grafted kidney function in recipients or in the outcomes of KT when donors and recipients were anesthetized with sevoflurane compared to desflurane.

\section{Materials and Methods}

After approval from the Institutional Review Board, we performed a retrospective review of the electronic medical records at our institution, of patients 20 years or older who had received a kidney transplant from a living donor from January 2006 to June 2009. We included pairs of donors and recipients where the recipient had been anesthetized with the same volatile anesthetics as the donor. Among the selected pairs, those with incomplete medical records or unstable hemodynamic events during the transplantation operation were excluded from the study (Fig. 1). We defined an unstable hemodynamic event as documentation of an intra-operative mean arterial pressure (MAP) of more than $30 \%$ below baseline or a blood oxygen saturation of less than $95 \%$. We ultimately identified 149 eligible pairs for this study. The pairs were divided into three groups: pairs anesthetized with sevoflurane ( $n=73$, Sevo group), pairs anesthetized with desflurane $(n=71$,
Des group), and pairs anaesthetized with isoflurane $(n=5)$. We excluded the pairs anesthetized with isoflurane because of the small sample size.

All donors and recipients were administered general anesthesia with mechanical ventilation. Induction of anesthesia was performed with an intravenous anesthetic (propofol or thiopental), an opioid (fentanyl or remifentanil), and a muscle relaxant (atracurium, vecuronium, or rocuronium). A balanced anesthetic technique was performed for maintenance anesthesia using a volatile anesthetic (sevoflurane or desflurane), an opioid (intermittent intravenous fentanyl or an intravenous infusion of remifentanil), and a muscle relaxant (intravenous infusion of atracurium). Soda lime was used as a carbon dioxide absorbent in all patients.

An immunosuppressed condition was achieved using the following regimen in both groups: induction of immunosuppression with baciliximab and methylprednisolone and maintenance with calcineurin inhibitor (cyclosporine or tacrolimus [FK 506]), methylprednisolone, and an antimetabolite (mycophenolic acid or mizoribine).

We analyzed the factors affecting grafted kidney function and compared them between the Sevo group and the Des group. These factors included the following: age, sex, weight, height, co-morbid disease, preoperative serum level of blood urea nitrogen (BUN) and creatinine (Cr), intra-operative intake/

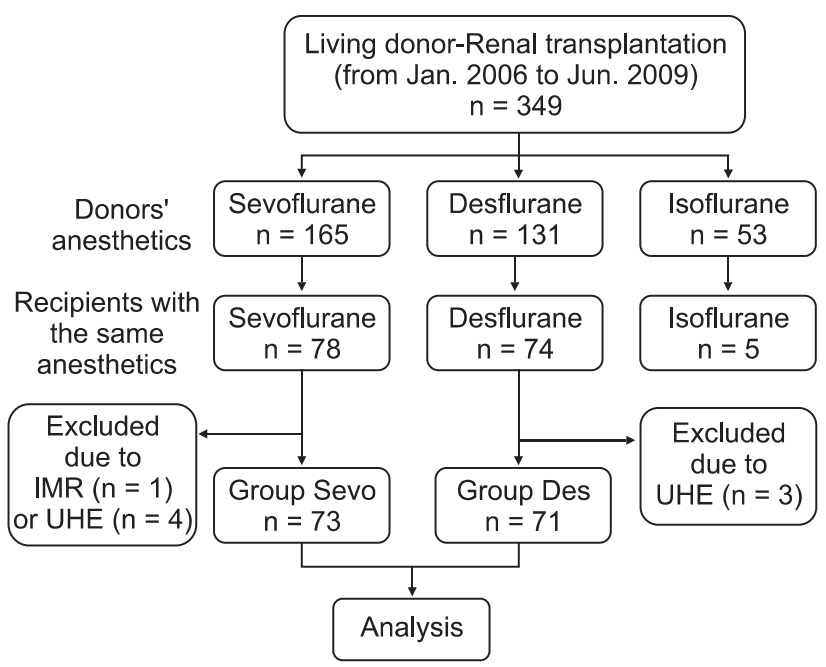

Fig. 1. Flowchart of data acquisition. Of the patients who underwent kidney transplantation from a living donor from January 2006 to June 2009, this study included donor-recipient pairs in which the recipient had been anesthetized with the same volatile anesthetic as the donor. We excluded the pairs in which isoflurane was used because of the small sample size. Finally, the enrolled pairs were divided into groups, the sevoflurane group ( $n=73$, Sevo group) and the desflurane group ( $\mathrm{n}=71$, Des group). IMR, incomplete medical record; UHE, unstable hemodynamic event during transplantation. 
output, total ischemic time, number of matched human leukocyte antigens (HLA)-A, B, and HLA-DR, and type of calcineurin inhibitor. Function of the grafted kidney was estimated by the serum level of BUN, Cr, and estimated glomerular filtration rate (eGFR) on postoperative day (POD) 1, 2, 4, and 7, at discharge, and at 12 months after transplantation. The eGFR was calculated using the MDRD (modification of diet in renal disease) equation. We also investigated the frequency of delayed graft function (DGF), treated acute rejection episodes (ARE), and graft loss within one year of transplantation. DGF was indicated in graft recipients who underwent dialysis during the first week after transplantation. ARE included both biopsy-proven acute rejection and clinically suspected acute rejection. We defined a graft loss as the initiation of long-term dialysis therapy after transplantation.

Data were analyzed using PASW Statistics 18.0 (SPSS Inc., Chicago, IL, USA). We used an independent t-test to compare the grafted kidney function between the two groups. We tested noninferiority for serum creatinine at discharge and 1 yr after KT. To test non-inferiority or equivalence, a margin of equivalence (the largest difference that is not of practical significance) must first be defined. Because the increase of $0.3 \mathrm{mg} / \mathrm{dl}$ of serum creatinine is attributed to 'risk' criteria according to the definition of acute kidney injury [12], a maximum difference of $0.3 \mathrm{mg} / \mathrm{dl}$ in the serum creatinine was considered as equivalence. The Fisher's exact test was used to compare the frequency of DGF between the two groups. A P value $<0.05$ was considered statistically significant.

\section{Results}

There were no significant differences in donor and recipient demographics or intraoperative data between the Sevo group and the Des group (Table 1).

Table 1. Demographic and Intra-operative Data of the Patients

\begin{tabular}{|c|c|c|c|}
\hline & $\begin{array}{c}\text { Sevo } \\
(\mathrm{n}=73)\end{array}$ & $\begin{array}{c}\text { Des } \\
(\mathrm{n}=71)\end{array}$ & $P$ value \\
\hline \multicolumn{4}{|l|}{ Transplant donors } \\
\hline $\operatorname{Sex}(M / F)$ & $41 / 32$ & $32 / 39$ & 0.18 \\
\hline Age & $40.5 \pm 10.7$ & $42.2 \pm 12.5$ & 0.36 \\
\hline Weight (kg) & $64.8 \pm 9.9$ & $62.9 \pm 9.9$ & 0.28 \\
\hline Height (cm) & $166.1 \pm 8.0$ & $164.4 \pm 8.5$ & 0.23 \\
\hline BUN (mg/dl) & $12.9 \pm 2.9$ & $12.2 \pm 3.2$ & 0.18 \\
\hline $\mathrm{Cr}(\mathrm{mg} / \mathrm{dl})$ & $0.89 \pm 0.13$ & $0.86 \pm 0.17$ & 0.20 \\
\hline Fluid intake (ml) & $2,622.2 \pm 855.0$ & $2,639.2 \pm 848.2$ & 0.91 \\
\hline Blood intake (ml) & $2.7 \pm 23.4$ & $2.8 \pm 23.7$ & 0.98 \\
\hline Urine output (ml) & $435.6 \pm 206.5$ & $404.9 \pm 230.5$ & 0.40 \\
\hline Blood loss (ml) & $183.2 \pm 163.4$ & $163.8 \pm 163.8$ & 0.48 \\
\hline \multicolumn{4}{|l|}{ Transplant recipients } \\
\hline $\operatorname{Sex}(M / F)$ & $41 / 32$ & $40 / 31$ & 0.98 \\
\hline Age & $42.5 \pm 11.3$ & $43.2 \pm 12.0$ & 0.71 \\
\hline Weight (kg) & $58.7 \pm 10.0$ & $58.8 \pm 12.4$ & 0.97 \\
\hline Height $(\mathrm{cm})$ & $164.9 \pm 7.6$ & $165.0 \pm 7.6$ & 0.95 \\
\hline \multicolumn{4}{|l|}{ Co-morbid disease } \\
\hline Hypertension & $64(87.7 \%)$ & $65(91.5 \%)$ & 0.59 \\
\hline Diabetes mellitus & $8(11.0 \%)$ & $9(12.7 \%)$ & 0.80 \\
\hline Hepatitis virus carrier & $4(5.5 \%)$ & $4(5.6 \%)$ & 1.0 \\
\hline Hypercholesterolemia & $9(12.3 \%)$ & $9(12.7 \%)$ & 1.0 \\
\hline BUN (mg/dl) & $45.5 \pm 20.4$ & $50.4 \pm 18.8$ & 0.16 \\
\hline $\mathrm{Cr}(\mathrm{mg} / \mathrm{dl})$ & $8.23 \pm 3.62$ & $8.53 \pm 3.33$ & 0.61 \\
\hline eGFR (ml/min) & $8.1 \pm 3.1$ & $8.1 \pm 3.8$ & 0.98 \\
\hline No. of HLA-A,B matched & $2(0-4)$ & $2(0-4)$ & 0.64 \\
\hline No. of HLA-DR matched & $1(0-2)$ & $1(0-2)$ & 0.55 \\
\hline PRA $>30 \%$ & $10(13.7 \%)$ & $8(11.3 \%)$ & 0.80 \\
\hline Total ischemic time (min) & $51.7 \pm 15.4$ & $55.8 \pm 16.0$ & 0.20 \\
\hline Fluid intake (ml) & $3,570.7 \pm 644.6$ & $3,825.4 \pm 1127.8$ & 0.11 \\
\hline Blood intake (ml) & $132.7 \pm 280.4$ & $184.6 \pm 268.4$ & 0.28 \\
\hline Urine output (ml) & $516.5 \pm 399.1$ & $652.1 \pm 466.6$ & 0.07 \\
\hline Blood loss (ml) & $317.0 \pm 273.5$ & $411.7 \pm 373.1$ & 0.08 \\
\hline $\begin{array}{c}\text { Type of calcineurin inhibitor } \\
\text { (Tacrolimus/Cyclosporine) }\end{array}$ & $55 / 18$ & $59 / 12$ & 0.31 \\
\hline
\end{tabular}

Data are mean \pm standard deviation, median (range), or number of patients (percent). HLA: human leukocyte antigen, eGFR: estimated glomerular filtration rate, PRA: panel reactive antibodies. 

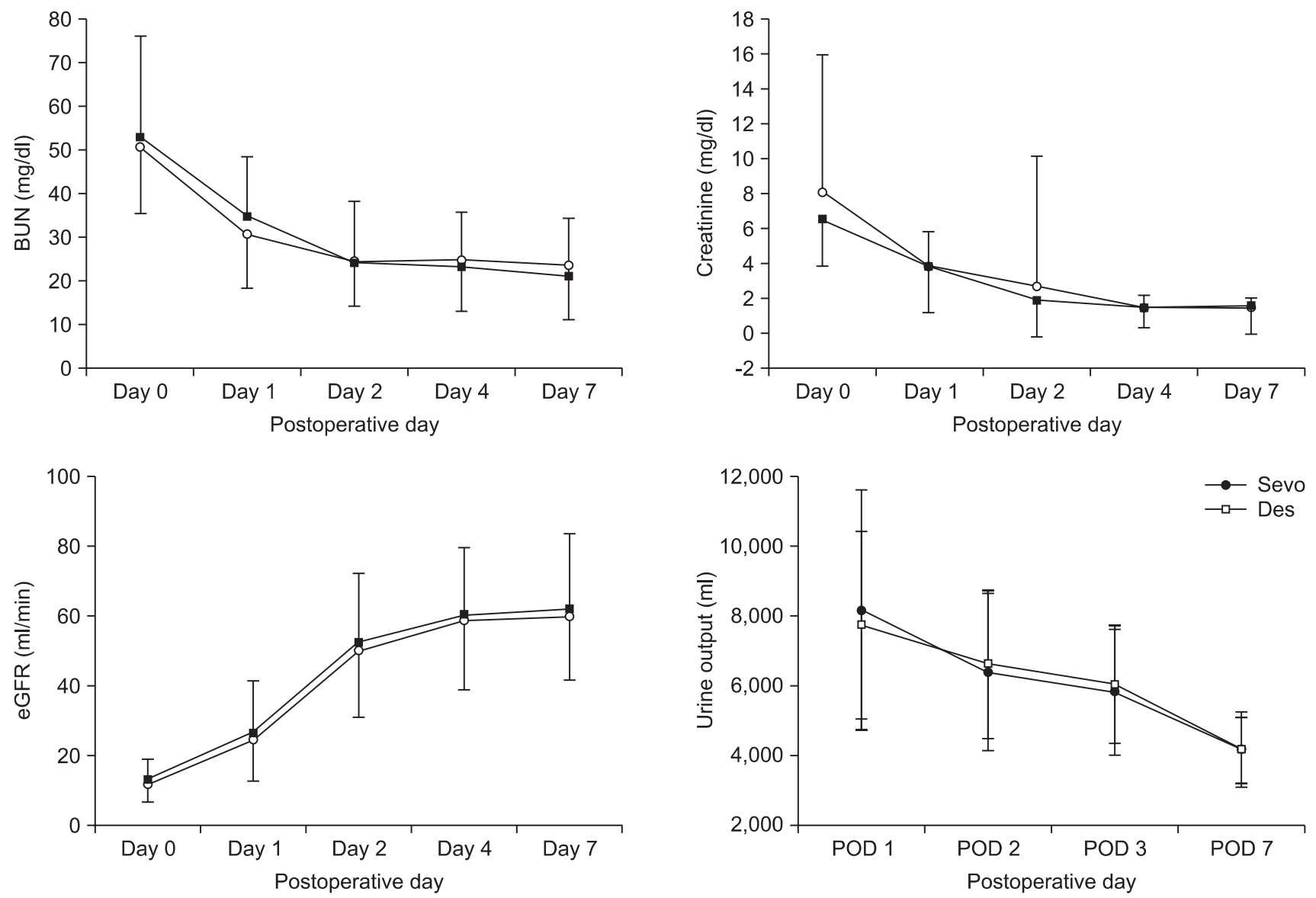

Fig. 2. Transplanted renal function in the immediate postoperative period. Changes in serum blood urea nitrogen (BUN), creatinine (Cr) level, estimated glomerular filtration rate (eGFR), and urine output in the Des group ( $\square$ ) and in the Sevo group (๑) during the immediate postoperative period. The changes in the serum concentrations of BUN, Cr, eGFR, and urine output of the recipients during the immediate postoperative period were comparable between the two groups.

Grafted kidney function during the immediate postoperative period was comparable between the two groups (Fig. 2). There were 2 patients with DGF in the Sevo group and 1 patient with DGF in the Des group; this difference was not statistically significant.

The duration of hospitalization and grafted kidney function at the time of discharge were comparable between the groups. The short-term outcomes of transplantation and transplanted renal function at 1 year after renal transplantation were also comparable between the two groups, as both groups showed no significant differences in the occurrence of ARE or graft loss for 1 year after transplantation (Table 2). The mean difference in serum creatinine and their $95 \%$ confidence interval (CI) are presented in Table 3. At the time of discharge, the 95\% CI (Sevo group minus Des group) fell within the margin of equivalence, and therefore the grafted kidney function on the discharge day between the groups was considered as equivalent. However, because the $95 \% \mathrm{CI}$ at the time of 1 year after surgery included
Table 2. Grafted Kidney Function and Outcomes at 1 Year after Transplantation

\begin{tabular}{lccl}
\hline & $\begin{array}{c}\text { Sevo } \\
(\mathrm{n}=73)\end{array}$ & $\begin{array}{c}\text { Des } \\
(\mathrm{n}=71)\end{array}$ & P value \\
\hline $\begin{array}{l}\text { Hospitalization days after surgery } \\
\text { No. of patients with DGF }\end{array}$ & $2(2.7 \pm 3.7$ & $20.3 \pm 28.8$ & 0.86 \\
On the day of discharge & & $1(1.4 \%)$ & 1.0 \\
$\quad$ BUN (mg/dl) & $21.3 \pm 5.9$ & $19.0 \pm 5.4$ & 0.18 \\
Cr (mg/dl) & $1.18 \pm 0.33$ & $1.21 \pm 0.39$ & 0.60 \\
eGFR (ml/min) & $65.9 \pm 14.8$ & $64.5 \pm 18.0$ & 0.38 \\
At 1 year after surgery & & & \\
BUN (mg/dl) & $17.8 \pm 5.5$ & $18.2 \pm 7.9$ & 0.68 \\
Cr (mg/dl) & $1.29 \pm 0.27$ & $1.45 \pm 0.95$ & 0.23 \\
$\quad$ eGFR (ml/min) & $62.7 \pm 15.0$ & $60.0 \pm 16.1$ & 0.30 \\
No. of AREs within 1 year & $17(23.3 \%)$ & $16(22.5 \%)$ & 0.91 \\
Graft loss within 1 year & 0 & 1 & 0.49 \\
\hline
\end{tabular}

All data are mean \pm standard deviation, number of episodes, or patients (percent). DGF: delayed graft function, eGFR: estimated glomerular filtration rate, ARE: acute rejection episode. 
Table 3. Mean Differences and Their 95\% Confidence Intervals for the Serum Creatinine (Sevo Group Minus Des Group) at the Time of Discharge and 1 Year after Surgery

\begin{tabular}{lcc}
\hline & Mean difference & 95\% confidence interval \\
\hline On the day of discharge & -0.03 & $-0.21-0.15$ \\
At 1 year after surgery & -0.16 & $-0.51-0.19$ \\
\hline
\end{tabular}

-0.3 , the grafted kidney function of the Sevo group at that time was considered as non-inferior, rather than equivalent, to that of the Des group.

\section{Discussion}

The results of this retrospective study provide no direct evidence that sevoflurane has deleterious effects on grafted kidney function compared to desflurane because there were no differences in postoperative serum creatinine, BUN, estimated GFR, or urine output between the sevoflurane and desflurane groups. Also, the outcomes of renal transplantation surgery at 1 year after surgery were comparable between the Sevo group and the Des group. Finally, we can conclude that sevoflurane does not adversely affect grafted renal function at the time of discharge and at 1 year after transplantation because the differences in creatinine between the two groups (Sevo group minus Des group) were less than $0.3 \mathrm{mg} / \mathrm{dl}$, which is the margin of equivalence.

A previous report has already demonstrated that sevoflurane has no detectable effect on the outcome of kidney transplantation [13]. This report assessed renal function in recipients anesthetized with sevoflurane compared to isoflurane, irrespective of the anesthetics used for the donors. However, in animal studies and a in handful of human studies, renal damage related to sevoflurane has been reported to be correlated with the exposure duration and the concentration of serum inorganic fluoride ion and inspired compound A [3]. As such, the transplanted kidneys may have been exposed to fluoride and compound $\mathrm{A}$ if the donors were anesthetized with sevoflurane. Therefore, if sevoflurane has an adverse effect on renal graft function, it should be more pronounced in grafted kidneys in which both the donor and recipient were anesthetized with sevoflurane.

Inorganic fluoride was suggested to be nephrotoxic in studies using methoxyflurane $[14,15]$. Although Higuchi et al. reported that elevated serum fluoride concentrations ( $>50 \mu \mathrm{mol} / \mathrm{L}$ ) secondary to sevoflurane may contribute to postoperative renal impairment [4], there was no such relationship between the serum fluoride concentrations and nephrotoxicity in the majority of other studies that used sevoflurane $[3,11]$. In contrast, compound A has been reported to be correlated with renal toxicity in several studies. In human volunteers, compound A, at concentrations ranging from 150 to 342 ppm-h after sevoflurane administration, induced transient abnormalities in urine biochemical markers [4,5]. Furthermore, compound A exposures of $240 \mathrm{ppm}$-h from sevoflurane resulted in albuminuria and increased excretion of $\alpha$-glutathione-Stransferase (a biochemical marker in urine) [7]. Additionally, surgical patients with low-flow sevoflurane anesthesia were exposed to compound A at 214 ppm-h and exhibited mild, transient proteinuria and increased excretion of N-acetyl$\beta$-D-glucosaminidase (a lysosomal enzyme located in the proximal renal tubule) [8]. Nevertheless, no studies have ever reported nephrotoxicity induced by sevoflurane that could be detected by routine clinical tests of renal function (serum BUN, Cr, or creatinine clearance). This suggests that nephrotoxicity secondary to sevoflurane may be too mild and transient to affect renal function. Additionally, there were also no detectable influences on renal function secondary to sevoflurane in patients with stable renal insufficiency $[16,17]$. Based on these studies, since 2006, sevoflurane has been administered to patients undergoing renal transplantation in our institution. However, grafted kidneys could be damaged by various factors during and even after renal transplantation. IR injury during the transplantation procedure may contribute to delayed graft function. This could subsequently increase allograft immunogenicity and increase the risk of acute rejection episodes, which could ultimately result in graft failure $[9,10]$. Furthermore, immunosuppressants such as cyclosporine or tacrolimus used after renal transplantation can cause nephrotoxicity, although they do result in decreased acute rejection rates and improved short-term outcomes [18]. These conditions in which the kidney grafts can be damaged during and after surgery are so unique that they are not observed in other types of surgeries. Renal damage secondary to sevoflurane may occur under these conditions, although nephrotoxicity attributable to sevoflurane is clinically insignificant in patients with stable renal insufficiency. Therefore, we need to confirm whether sevoflurane can safely be used in patients undergoing renal transplantation.

There is yet another issue regarding sevoflurane and renal function in renal transplantation. There have been several reports suggesting the protective effects of volatile anesthetics against renal IR injury [19-21]. These protective effects have not been definitively established; however, these reports suggest that volatile anesthetics may actually have a positive effect on the human kidney. In clinical practice, potential evidence for renal protection by volatile anesthetics was illustrated in a recent study by Julier et al. [22]. Their study showed that sevoflurane pretreatment before cardiopulmonary bypass for coronary artery bypass grafting surgery markedly improved 
postoperative GFR as determined by the plasma concentration of cystatin C. Therefore, sevoflurane may confer positive effects on renal graft function by reducing IR injury.

There are several limitations to this study. First, we could not assess the levels of inorganic fluoride and compound A. Therefore, we could not confirm whether these affect the grafted kidney function. In addition, we evaluated the function of the grafted kidney, not the severity of damage related to sevoflurane. To clarify the nephrotoxicity of sevoflurane, we need to assess the renal injury using a novel biomarker such as neutrophil gelatinase-associated lipocalin (NGAL). NGAL has emerged as a new diagnostic tool for acute kidney injury [23]. In kidney transplant patients, NGAL not only predicts DGF $[24,25]$, but also correlates with grafted kidney function $[26,27]$. Second, we were unable to confirm whether or not sevoflurane had an effect on grafted renal function in the immediate posttransplantation period. However, we showed that there was no deterioration in grafted renal function in the Sevo group at the time of discharge and 1 year after renal transplantation. Therefore, we believe that sevoflurane can safely be used in patients undergoing renal transplantation.

In conclusion, sevoflurane has no adverse effects on renal graft function or on the short-term outcome of renal transplantation compared to desflurane.

\section{References}

1. Holaday DA, Smith FR. Clinical characteristics and biotransformation of sevoflurane in healthy human volunteers. Anesthesiology 1981; 54: 100-6

2. Gonsowski CT, Laster MJ, Eger EI 2nd, Ferrell LD, Kerschmann RL. Toxicity of compound A in rats. Effect of a 3-hour administration. Anesthesiology 1994; 80: 556-65.

3. Gentz BA, Malan TP Jr. Renal toxicity with sevoflurane: a storm in a teacup? Drugs 2001; 61: 2155-62.

4. Higuchi H, Sumikura H, Sumita S, Arimura S, Takamatsu F, Kanno $\mathrm{M}$, et al. Renal function in patients with high serum fluoride concentrations after prolonged sevoflurane anesthesia. Anesthesiology 1995; 83: 449-58.

5. Eger EI 2nd, Koblin DD, Bowland T, Ionescu P, Laster MJ, Fang Z, et al. Nephrotoxicity of sevoflurane versus desflurane anesthesia in volunteers. Anesth Analg 1997; 84: 160-8.

6. Eger EI 2nd, Gong D, Koblin DD, Bowland T, Ionescu P, Laster MJ, et al. Dose-related biochemical markers of renal injury after sevoflurane versus desflurane anesthesia in volunteers. Anesth Analg 1997; 85: 1154-63.

7. Goldberg ME, Cantillo J, Gratz I, Deal E, Vekeman D, McDougall R, et al. Dose of compound A, not sevoflurane, determines changes in the biochemical markers of renal injury in healthy volunteers. Anesth Analg 1999; 88: 437-45.

8. Higuchi H, Sumita S, Wada H, Ura T, Ikemoto T, Nakai T, et al. Effects of sevoflurane and isoflurane on renal function and on possible markers of nephrotoxicity. Anesthesiology 1998; 89: 307-22.

9. Perico N, Cattaneo D, Sayegh MH, Remuzzi G. Delayed graft function in kidney transplantation. Lancet 2004; 364: 1814-27.

10. Kosieradzki M, Rowinski W. Ischemia/reperfusion injury in kidney transplantation: mechanisms and prevention. Transplant Proc 2008; 40: 3279-88.

11. Artru AA. Renal effects of sevoflurane during conditions of possible increased risk. J Clin Anesth 1998; 10: 531-8.

12. Kellum JA, Bellomo R, Ronco C. Definition and classification of acute kidney injury. Nephron Clin Pract 2008; 109:c182-7.

13. Teixeira S, Costa G, Costa F, da Silva Viana J, Mota A. Sevoflurane versus isoflurane: does it matter in renal transplantation? Transplant Proc 2007; 39: 2486-8.

14. Cousins MJ, Mazze RI. Methoxyflurane nephrotoxicity. A study of dose response in man. JAMA 1973; 225: 1611-6.

15. Cousins MJ, Mazze RI, Kosek JC, Hitt BA, Love FV. The etiology of methoxyflurane nephrotoxicity. J Pharmacol Exp Ther 1974; 190: 530-41.

16. Conzen PF, Kharasch ED, Czerner SF, Artru AA, Reichle FM, Michalowski $\mathrm{P}$, et al. Low-flow sevoflurane compared with low-flow isoflurane anesthesia in patients with stable renal insufficiency. Anesthesiology 2002; 97: 578-84.

17. Conzen PF, Nuscheler M, Melotte A, Verhaegen M, Leupolt T, Van Aken $\mathrm{H}$, et al. Renal function and serum fluoride concentrations in patients with stable renal insufficiency after anesthesia with sevoflurane or enflurane. Anesth Analg 1995; 81: 569-75.

18. Yabu JM, Vincenti F. Kidney transplantation: the ideal immunosuppression regimen. Adv Chronic Kidney Dis 2009; 16: 226-33.

19. Lee HT, Ota-Setlik A, Fu Y, Nasr SH, Emala CW. Differential protective effects of volatile anesthetics against renal ischemiareperfusion injury in vivo. Anesthesiology 2004; 101: 1313-24.

20. Hashiguchi H, Morooka H, Miyoshi H, Matsumoto M, Koji T, Sumikawa K. Isoflurane protects renal function against ischemia and reperfusion through inhibition of protein kinases, JNK and ERK. Anesth Analg 2005; 101: 1584-9.

21. Obal D, Rascher K, Favoccia C, Dettwiler S, Schlack W. Post-conditioning by a short administration of desflurane reduced renal reperfusion injury after differing of ischaemia times in rats. $\mathrm{Br} \mathrm{J}$ Anaesth 2006; 97: 783-91.

22. Julier K, da Silva R, Garcia C, Bestmann L, Frascarolo P, Zollinger $\mathrm{A}$, et al. Preconditioning by sevoflurane decreases biochemical markers for myocardial and renal dysfunction in coronary artery bypass graft surgery: a double-blinded, placebo-controlled, multicenter study. Anesthesiology 2003; 98: 1315-27.

23. Haase M, Bellomo R, Devarajan P, Schlattmann P, Haase-Fielitz A. Accuracy of neutrophil gelatinase-associated lipocalin (NGAL) in diagnosis and prognosis in acute kidney injury: a systematic review and meta-analysis. Am J Kidney Dis 2009; 54: 1012-24.

24. Parikh CR, Jani A, Mishra J, Ma Q, Kelly C, Barasch J, et al. Urine NGAL and IL-18 are predictive biomarkers for delayed graft function following kidney transplantation. Am J Transplant 2006; 6: 1639-45.

25. Hall IE, Yarlagadda SG, Coca SG, Wang Z, Doshi M, Devarajan P, et al. IL-18 and urinary NGAL predict dialysis and graft recovery after kidney transplantation. J Am Soc Nephrol 2010; 21: 189-97.

26. Hollmen ME, Kyllonen LE, Inkinen KA, Lalla ML, Salmela KT. Urine neutrophil gelatinase-associated lipocalin is a marker of graft 
recovery after kidney transplantation. Kidney Int 2011; 79: 89-98.

27. Lebkowska U, Malyszko J, Lebkowska A, Koc-Zorawska E, Lebkowski W, Malyszko JS, et al. Neutrophil gelatinase-associated lipocalin and cystatin C could predict renal outcome in patients undergoing kidney allograft transplantation: a prospective study. Transplant Proc 2009; 41: 154-7. 